\title{
Nanometric transverse displacement metrology in range of hundreds of micrometers with polarization- encoded metasurface
}

\section{Haofeng Zang}

University of Science and Technology of China

\section{Zheng Xi}

University of Science and Technology of China

Zhiyu Zhang

University of Science and Technology of China

Yonghua Lu ( $\square$ yhlu@ustc.edu.cn )

University of Science and Technology of China

Pei Wang

Institute of Photonics, Department of Optics and Optical Engineering, University of Science and

Technology of China

Article

Keywords:

Posted Date: January 6th, 2022

DOI: https://doi.org/10.21203/rs.3.rs-1152151/v1

License: (c) (1) This work is licensed under a Creative Commons Attribution 4.0 International License.

Read Full License 


\section{Abstract}

A long range, high precision and compact transverse displacement metrology method is of crucial importance in many research areas. We propose and experimentally demonstrate the first prototype polarization-encoded metasurface for ultrasensitive transverse displacement metrology. The transverse displacement of the metasurface is encoded into the polarization direction of the outgoing light via the Pancharatnam-Berry phase. By measuring the output light polarization direction, the metasurface's position can be readout directly according to the Malus law. We experimentally demonstrate nanometer displacement resolution with the uncertainty on the order of $100 \mathrm{pm}$ for a large measurement range of $200 \mu \mathrm{m}$ with the total area of the metasurface being within $900 \mu \mathrm{m} \times 900 \mu \mathrm{m}$. The measurement range can be extended further using a larger metasurface. Our work largely broadens the existing application areas of metasurface and opens new avenue of applying metasurface in the field of ultrasensitive optical transverse displacement metrology.

\section{Full Text}

This preprint is available for download as a PDF.

\section{Supplementary Files}

This is a list of supplementary files associated with this preprint. Click to download.

- Supplementary.docx 